\title{
Conjecturing constant cross-ratio loci of a pencil in $E_{2}$
}

\author{
G.L.Narasimham \\ Ex Indian Space Research Organisation
}

\begin{abstract}
It is sought to find a locus of a variable point having constant cross-ratio of a pencil of rays emanating from it and connecting to four fixed points in order to relate its cross-ratio to a conic property.
\end{abstract}

Introduction: Five points determine a conic. If four points are fixed, invariant properties of a conic can be defined by inclusion of a fifth point to the point set. At the same time for all transversal $\mathrm{T}$ intersections, cross-ratio of a pencil arising from four rays of these four fixed points is a known constant even when $\mathrm{A}, \mathrm{B}, \mathrm{C}$ and $\mathrm{D}$ are not collinear.

Discussion: In this short note, known dimensionless conic eccentricity is conjectured to depend functionally on the dimensionless cross ratio of the four fixed points with respect to the moving fifth point vertex because cross-ratio (here abbreviated XR) is most cited projective invariant property of the four ray pencil.

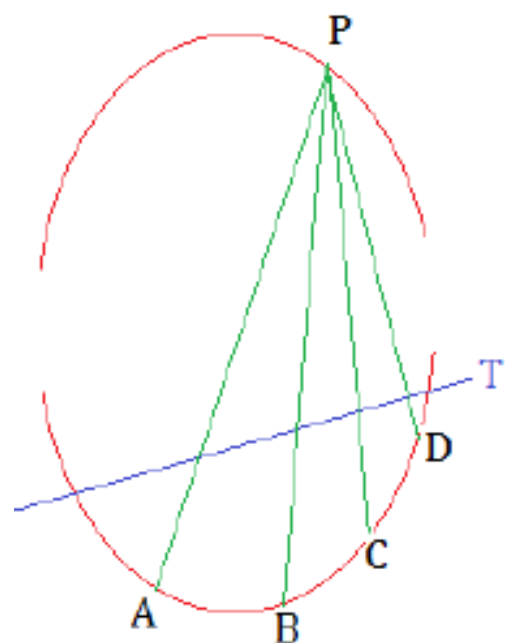

Fig 1. 4 Ray Pencil

There are two definitions of XR: One for four collinear points and another for four concurrent lines. The latter Euclidean form/ definition of co-planar concurrent lines rather than classical definition of collinear four point set is used here for detecting a constant XR curve. It is expressed in terms of sines of angles included amongst a concurrent ray set. If the variable point $\mathrm{P}(\mathrm{x}, \mathrm{y})$ is connected to four fixed points A (the origin, say), B, C and D, then

CrossRatio of 4 ray pencil $=\frac{\sin (A P C) \sin (B P D)}{\sin \left(B^{\prime} C^{\circ}\right) \sin (A P D)}$

The above can be greatly simplified if a rectangular region is chosen to yield a fourth order polynomial because it can be factored into two second order conics, which is expected from a double or cross-ratio definition. Its graphical plots enabled a visual recognition of locus

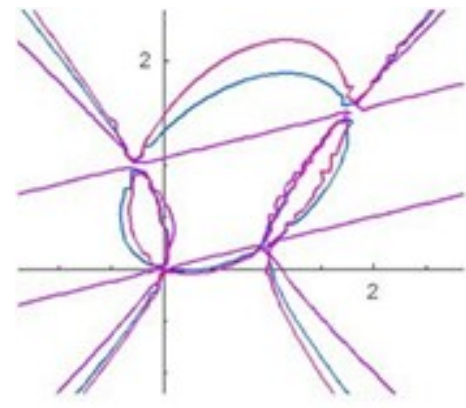

Fig 2. Plots of constant cross-ratio unmistakably as ellipses, hyperbola nappes and pairs of straight lines for various XR. The author has verified validity of this conjecture as follows: For simplicity and without loss of generality (since XR is invariant not only by simple axes translation or rotation), a central conic locus is assumed with axes parallel to $\mathrm{X}$ and $\mathrm{y}$ - axes, shifted topass through four corners of a rectangle including the origin: $(0,0),(2 \mathrm{~h}, 0),(2 \mathrm{~h}, 2 \mathrm{k})$ and $(0,2 \mathrm{k})$. Equation (1) after algebraic simplification has been analytically factored into two conics, a primary and a secondary: 


$$
\begin{aligned}
& \frac{(x-h)^{2}}{a^{2}}+\frac{(y-k)^{2}}{b^{2}}-1 \\
& \frac{\left(\frac{x}{h}-\frac{y}{k}\right)\left(\frac{x}{h}+\frac{y}{k}-2\right)}{(x / h)(2-x / h)}= \pm X R
\end{aligned}
$$

(2a) and

The first involves XR in the left hand side of Equation and the second on the right. The required conics ( 2 b) may also be termed conjugate. XR can be positive or negative. Primary with $\mathrm{XR}>0$ is an ellipse, secondary is a hyperbola for $\mathrm{XR}<0$ (since one angle subtended is $>\pi$ ) and a pair of straight lines for $\mathrm{XR}=0$.

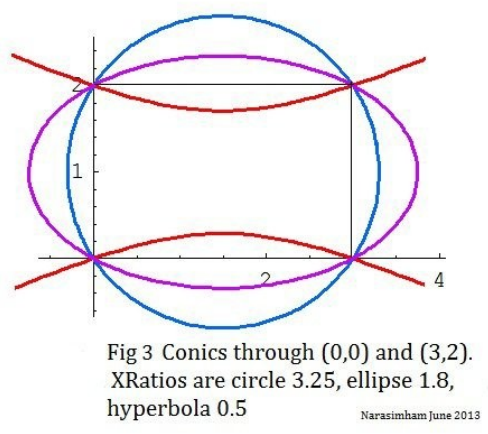

For the primary conic: Satisfaction of the four point boundary conditions imposes a condition enabling a key definition for semi axes $a$ and $b$.

$a= \pm h \frac{b}{\sqrt{k^{2}+b^{2}}}$

The condition leads to relations for the major and minor axes:

$\frac{a}{h}=\sqrt{X R /(X R-1)} ; \frac{b}{k}=\sqrt{X R}$;

The plots when superimposed have an exact graphics tally also, confirming the expressions and conjecture. The cases for 3:2 rectangle corners inclusive conics have also been checked by direct

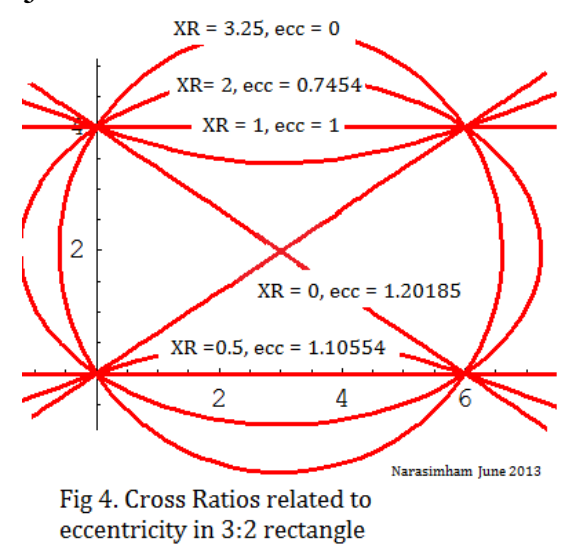

$X R=\left(\frac{a}{b}\right)^{2}\left(1-\epsilon^{2}\right)+1=\left(\frac{h b}{k a}\right)^{2}+1$
CAS geometric constriction, to verify that Equations (2), (4) satisfy Equation (1) directly.

Some examples are plotted Figs 4 and 5. The particular case of $\mathrm{P}$ locus as a circle that includes four corners of a square is interesting. It has $\mathrm{XR}=2$.

If the locus passes through them as a pair of straight lines, $\mathrm{XR}=0$, inclined at $\arctan (\mathrm{h} / \mathrm{k})$. For all conics passing through rectangle corners, major, minor axes can be related to XR and eccentricity as follows:

Square of minor axis and XR is negative for a hyperbola, positive for ellipse.

Motivation: for obtaining these relations is felt lack of immediately available examples in articles and talks on projective geometry. In everyday experience, say if a commodity (locus curve) is known to have a fixed price (constant XR value) available in a certain departmental stores (a set of definable curves) but is not known what that item is, then there is no demand (for lack of its definition or identification). Lack of assertion, that a conic is a unique example of constant XR concurrent pencil apex locus ... appears to cloud the main concept.

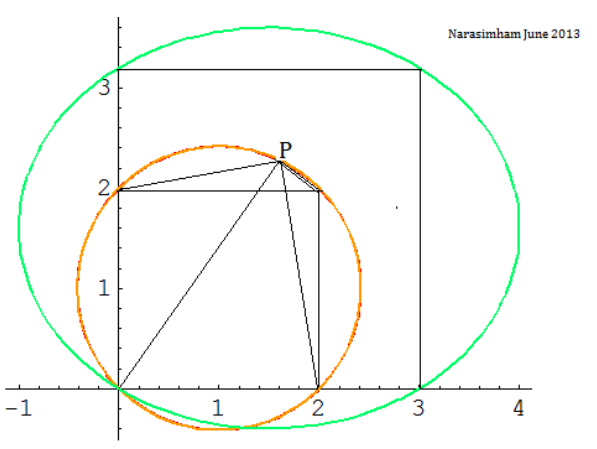

Fig 5. A circle through four corners of any $(2 \times 2$ here) square has $\mathrm{XR}=2$. An ellipse of proportions $\left.2^{*}(\mathrm{a}, \mathrm{b}, \mathrm{c})\right)=(5,4,3)$ passing through corners of $(3$ $X$ 3.2) rectangle has an $X R=1.5625$ 
Chasles theorem gives a general indication of a conic as the needed locus in a general. Perhaps it does not mention the existence of the conjugate/associated conic for negative $\mathrm{XR}$, seen from limited literature available to the author.

Fig 6 depicts a surface assembled of constant XR loci, along with its conjugate at each height when sectioned.
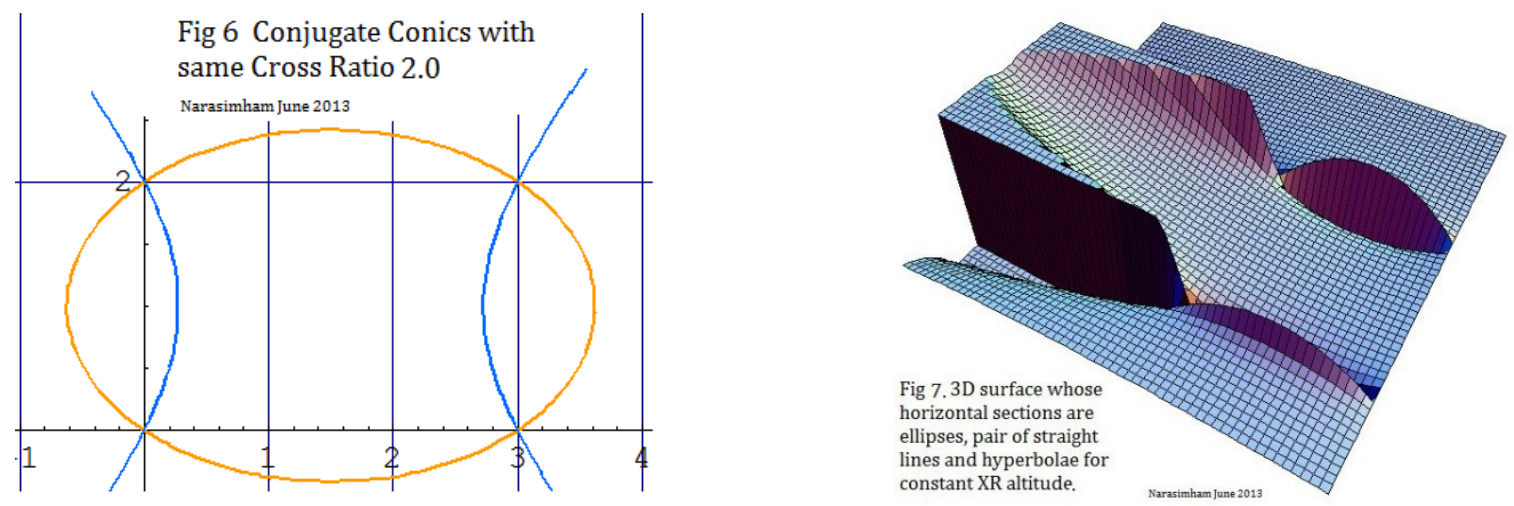

Conclusion: The relations given here furnish particular simple cases around a rectangle. They are simpler than conics in classical form: $\mathrm{a} \mathrm{x}^{\wedge} 2+2 \mathrm{~h} \mathrm{x} \mathrm{y}+\mathrm{b} \mathrm{y}^{\wedge} 2+2 \mathrm{f} \mathrm{x}+2 \mathrm{~g} \mathrm{y}=1$, circumscribing the four points or describing hyperbolic arcs/ nappes through them as in Fig 2. A conic pair in Equations (2) is the only constant XR in E2, up to Euclidean motions. The particular cases could find ready applications in projective, algebraic or hyperbolic geometries. 